\title{
Dynamic snap-through of a laterally loaded arch under prescribed end motion
}

\author{
Jian-San Lin, Jen-San Chen * \\ Department of Mechanical Engineering, National Taiwan University, Taipei 10617, Taiwan \\ Received 1 November 2002; received in revised form 5 March 2003
}

\begin{abstract}
Emphasis of this paper is placed on finding out whether dynamic snap-through will occur when the laterally loaded arch is under prescribed end motion with constant speed. The first harmonic component $q_{1} \sin \xi$ of the lateral load is assumed to be the dominant one, while the effects of higher components such as $q_{2} \sin 2 \xi$ are also discussed in detail. It is found that dynamic snap-through may occur in either stretching or compressing process when the end speed of the loaded arch is in certain range. The dangerous zones for dynamic snap-through can be determined by comparing the energy barrier and the total energy gained by the arch when the end is moved with infinitely large speed. Generally speaking, for a specified $q_{1}$ it is easier for the arch under prescribed end motion to snap dynamically if $q_{2} \neq 0$.
\end{abstract}

(C) 2003 Elsevier Ltd. All rights reserved.

Keywords: Dynamic snap-through; Shallow arch; Prescribed end motion

\section{Introduction}

When a lateral load tending to flatten a sinusoidal arch is applied quasi-statically, the axial thrust in the arch will develop and grow due to the immovability of the ends. For a specified arch shape the arch may undergo snap-through buckling at certain critical load. Early classical investigation can be found in Timoshenko (1935), Fung and Kaplan (1952), Gjelsvik and Bonder (1962), Onat and Shu (1962), Franciosi et al. (1964), Schreyer and Masur (1966), Lee and Murphy (1968), and Simitses (1973). Experimental results have been reported by Roorda (1965). In the case when the lateral load is applied dynamically instead of in the quasi-static manner, the critical load will be different from the one predicted statically, see Hoff and Bruce, 1954; Humphreys, 1966; Lock, 1966; Hsu, 1967, 1968; Hsu et al., 1968; Patricio et al., 1998; Xu et al., 2002. A comprehensive review on the dynamic instability of shallow arches can be found in the book by Simitses (1990), which also includes other structures such as shallow spherical cap and thin cylindrical shell.

Generally speaking, the methodologies used in estimating dynamic critical loads of elastic structures can be classified in two groups (Simitses, 1990). The first approach is to solve the equations of motion

\footnotetext{
${ }^{*}$ Corresponding author. Tel.: +886-2-2366-1734; fax: +886-2-2363-1755.

E-mail address: jschen@ccms.ntu.edu.tw (J.-S. Chen).
} 
numerically to obtain the system response. The load parameter at which there exists a large change in the response is called critical, for example, see Budiansky and Roth (1962) on spherical cap, and Kistler and Waas (1998, 1999) on cylindrical panel. In Kistler and Waas' works, the effects of different in-plane boundary conditions on the limit points of the response are also considered. This direct approach requires large amount of calculation in a wide parameter range. The second approach is to study the total energy and the phase plane of the system. By this method sufficient conditions for dynamic stability and instability may be established, for example, see Hsu $(1967,1968)$. A combination of both approaches would prove useful in practical engineering applications.

In this paper we investigate a new elastic stability problem which involves a loaded arch under prescribed end motion. At time $t=0$ we assume that one end of the loaded arch starts to move to a new position with constant speed, while the other end remains fixed in space. We wish to find out whether it is possible for the arch to be snapped to the other side dynamically and stay there if damping is present. This problem not only is new from the academic point of view, it may have practical application as well. Consider an arch structure designed and constructed to be in a stable equilibrium position. In the case when certain unexpected disturbance (for instance, in an earthquake or landslide) occurs such that the distance between the two ends of the arch changes, it is important to predict whether the structure still holds or not.

We assume that the laterally loaded arch is in a stable equilibrium configuration before one of the two ends is moved as prescribed. The magnitude and direction of the lateral load remain unchanged when the end motion is in progress. We first investigate the quasi-static case when the end speed is negligible. For a specified arch rise parameter and a moving distance of the end, we will determine all possible equilibrium configurations and their stability properties. If for a specified moving distance there are more than one stable equilibrium configurations, it is possible for the arch to jump from one stable configuration to the other and stay there if some damping mechanism is provided. In general it is very difficult to determine the necessary and sufficient condition for dynamic snap-through to occur. However, it is possible to propose a sufficient condition against dynamic snap-through. Effects of various disturbances on the response of the arch under prescribed end motion are also studied in detail in this paper, which include (1) the non-ideal initial conditions at the instance when the end starts to move, and (2) the imperfect distribution of the lateral loads.

\section{Equations of motion}

Fig. 1 shows an elastic shallow arch with the two pinned ends being separated originally by a distance $L$. The initial shape of the unloaded arch is $y_{0}(x)$. The arch is subjected to lateral loading $Q^{*}(x)$ and stays in a stable equilibrium configuration. At time $t=0$, the end at $x=L$ starts to move a distance $\delta$ with constant speed $c^{*} . \delta<0$ means that the arch is compressed. The equation of motion of the arch can be written as

$$
\rho A y_{, t t}=-E I\left(y-y_{0}\right)_{, x x x}+p^{*} y_{, x x}-Q^{*} \text {. }
$$

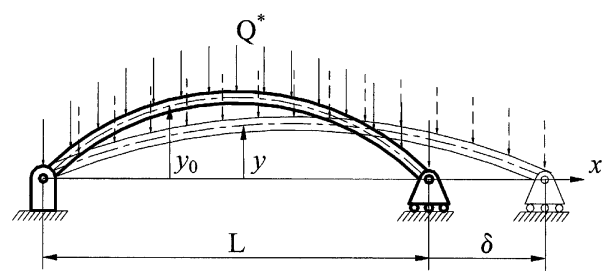

Fig. 1. Schematic diagram of a laterally loaded shallow arch under prescribed end motion. 
The parameters $E, \rho, A$, and $I$ are Young's modulus, mass density, area, and area moment of inertia of the cross-section of the arch. $p^{*}$ is the axial force,

$$
p^{*}(t)=\frac{A E}{L}\left[\delta(t)+\frac{1}{2} \int_{0}^{L}\left(y_{, x}^{2}-y_{0, x}^{2}\right) \mathrm{d} x\right] .
$$

In writing Eqs. (1) and (2) we neglect the inertial effect in the axial direction. The boundary conditions for $y$ at $x=0$ and $L$ are

$$
y(0)-y_{0}(0)=y_{, x x}(0)-y_{0, x x}(0)=y(L)-y_{0}(L)=y_{, x x}(L)-y_{0, x x}(L)=0 .
$$

Eqs. (1) and (2) can be non-dimensionalized to the forms

$$
\begin{aligned}
& u_{, \tau \tau}=-\left(u-u_{0}\right)_{, \xi \xi \xi \xi}+p u_{, \xi \xi}-Q, \\
& p=e+\frac{1}{2 \pi} \int_{0}^{\pi}\left(u_{, \xi}^{2}-u_{0, \xi}^{2}\right) \mathrm{d} \xi,
\end{aligned}
$$

where

$$
u=\frac{y}{r}, \quad u_{0}=\frac{y_{0}}{r}, \quad \xi=\frac{\pi x}{L}, \quad \tau=\frac{\pi^{2} t}{L^{2}} \sqrt{\frac{E I}{A \rho}}, \quad p=\frac{p^{*} L^{2}}{\pi^{2} E I}, \quad e=\frac{L \delta}{\pi^{2} r^{2}}, \quad Q=\frac{Q^{*} L^{4}}{\pi^{4} E I r} .
$$

$r$ is the radius of gyration of the cross-section. $p=1$ corresponds to the Euler buckling load for a perfectly straight simply supported beam. The transverse loading $Q(\xi)$ is assumed to be distributed in the form

$$
Q(\xi)=\sum_{j=1}^{\infty} q_{j} \sin j \xi
$$

It is noted that $q_{1}$ is positive when this harmonic component points downward in Fig. 1. The initial shape of the arch before the lateral load is applied is assumed to be in the form

$$
u_{0}=h \sin \xi .
$$

$h$ is the rise parameter of the arch.

It is assumed that the shape of the loaded arch after being stretched or compressed can be expanded as

$$
u(\tau, \xi)=u_{0}+\sum_{n=1}^{\infty} \alpha_{n}(\tau) \sin n \xi .
$$

After substituting Eqs. (7)-(9) into (4) and (5) we obtain the equations governing $\alpha_{n}$,

$$
\begin{aligned}
& \ddot{\alpha}_{1}=-\alpha_{1}-(G+e)\left(h+\alpha_{1}\right)-q_{1}, \\
& \ddot{\alpha}_{n}=-n^{4} \alpha_{n}-n^{2}(G+e) \alpha_{n}-q_{n}, \quad n=2,3, \ldots
\end{aligned}
$$

where

$$
\begin{aligned}
& G=\frac{1}{4} \sum_{k=1}^{\infty} k^{2} \alpha_{k}^{2}+\frac{h}{2} \alpha_{1}, \\
& e(\tau)=c \tau .
\end{aligned}
$$


The dimensionless speed $c$ is related to $c^{*}$ by

$$
c=\frac{L^{3}}{\pi^{4} r^{3}} \frac{c^{*}}{c_{1}},
$$

where $c_{1}$ is the longitudinal wave speed of the arch. The overhead dots in Eqs. (10) and (11) represent differentiation with respect to $\tau$. The axial force $p$ in Eq. (5) can be calculated as

$$
p=G+e .
$$

For the case without any initial disturbance, the initial conditions for Eqs. (10) and (11) are

$$
\begin{aligned}
& \alpha_{n}(0)=\alpha_{n}^{0}, \quad n=1,2,3, \ldots \\
& \dot{\alpha}_{n}(0)=0, \quad n=1,2,3, \ldots
\end{aligned}
$$

$\alpha_{n}^{0}$ corresponds to the equilibrium configuration of the arch under lateral load $Q$ before any end motion.

\section{Equilibrium configurations for $Q=q_{1} \sin \xi$}

We first consider the case when the lateral load is distributed in a manner such that $q_{1} \neq 0$ and $q_{i}=0$ for $i \neq 1$. In the case when the speed $c$ is small the acceleration terms in Eqs. (10) and (11) can be neglected. From this condition we can examine the equilibrium configurations for various values of $e$. Two types of solutions are possible, i.e., one-mode and two-mode solutions.

One-mode solution $u=u_{0}+\alpha_{1} \sin \xi$ :

$\alpha_{1}$ satisfies the following cubic equation:

$$
-e\left(\alpha_{1}+h\right)=\frac{\alpha_{1}}{4}\left(\alpha_{1}^{2}+3 h \alpha_{1}+2 h^{2}+4\right)+q_{1} .
$$

We first define the parameter $e_{1}$ as

$$
e_{1}-e_{\mathrm{T}}=-\frac{3}{4}\left(2\left|h-q_{1}\right|\right)^{2 / 3}
$$

where

$$
e_{\mathrm{T}}=\frac{h^{2}}{4}-1 \text {. }
$$

After defining $e_{1}$ we can make the following observations:

Case (1): $q_{1}<h$

If $e>e_{1}$, then there is only one equilibrium configuration $P_{0}$.

If $e<e_{1}$, then there are three equilibrium configurations $P_{0}, P_{1}^{+}$, and $P_{1}^{-}$, where

$$
\alpha_{1}\left(P_{0}\right)>-h>\alpha_{1}\left(P_{1}^{+}\right)>-h-\left[2\left(h-q_{1}\right)\right]^{1 / 3}>\alpha_{1}\left(P_{1}^{-}\right) .
$$

Case (2): $q_{1}>h$

If $e>e_{1}$, then there is only one equilibrium configuration $P_{1}^{-}$. where

If $e<e_{1}$, then there are three equilibrium configurations $P_{0}, P_{1}^{+}$, and $P_{1}^{-}$,

$$
\alpha_{1}\left(P_{0}\right)>-h-\left[2\left(h-q_{1}\right)\right]^{1 / 3}>\alpha_{1}\left(P_{1}^{+}\right)>-h>\alpha_{1}\left(P_{1}^{-}\right) .
$$


In the special case when $q_{1}=h$ and $e>e_{1}$, then the $P_{0}$ solution in Case (1) approaches the $P_{1}^{-}$solution in Case (2) and the only equilibrium configuration of the deformed arch is in the shape of a straight line. This can be readily verified as the lateral load $q_{1}=h$ always admits a root $\alpha_{1}=-h$ in Eq. (18).

Two-mode solution $u=u_{0}+\alpha_{1} \sin \xi+\alpha_{j} \sin j \xi$ :

For this case the solutions can be written explicitly,

$$
\begin{aligned}
& \alpha_{1}=\frac{q_{1}-j^{2} h}{j^{2}-1}, \\
& \alpha_{j}= \pm \frac{2}{j} \sqrt{e_{j}-e}, \quad j \neq 1,
\end{aligned}
$$

where

$$
e_{j}-e_{\mathrm{T}}=1-j^{2}-\frac{\left(h-q_{1}\right)^{2}}{4\left(j^{2}-1\right)^{2}} .
$$

These configurations are denoted by $P_{1 j}^{+}$and $P_{1 j}^{-}$, which exist only when

$$
e<e_{j}
$$

It is noted that $\alpha_{1}\left(P_{1 j}^{ \pm}\right)$is independent of $e$.

\section{Stability properties}

The method of determining the stability of the above equilibrium configurations has been laid out explicitly in Lin (2002), and the conclusions are summarized in the following.

One-mode solutions:

Case (1) $q_{1}<h$ : $P_{0}$ is always stable. $P_{1}^{+}$is always unstable. If $\left|q_{1}-h\right| \leqslant 3 \sqrt{6}$, then $P_{1}^{-}$is stable if and only if $e<e_{1}$. If $\left|q_{1}-h\right|>3 \sqrt{6}$ then $P_{1}^{-}$is stable if and only if $e<e_{2}$.

Case (2) $q_{1}>h: P_{1}^{-}$is always stable. $P_{1}^{+}$is always unstable. If $\left|q_{1}-h\right| \leqslant 3 \sqrt{6}$, then $P_{0}$ is stable if and only if $e<e_{1}$. If $\left|q_{1}-h\right|>3 \sqrt{6}$ then $P_{0}$ is stable if and only if $e<e_{2}$.

Two-mode solutions: $P_{1 j}^{+}$and $P_{1 j}^{-}$are always unstable.

Fig. 2 shows the bifurcation set using $q_{1}-h$ and $e-e_{\mathrm{T}}$ as control parameters. The tip T of the $e_{1}-e_{\mathrm{T}}$ curve is always at the origin. Fig. 2 is divided into nine regions by the solid $e_{j}-e_{\mathrm{T}}$ curves. The equilibrium configurations in each region are listed in Table 1, in which the stable configurations are labeled with boldtype symbols.

It is noted that the $e_{2}-e_{\mathrm{T}}$ curve touches $e_{1}-e_{\mathrm{T}}$ curve when $q_{1}-h= \pm 3 \sqrt{6}$ and $e-e_{\mathrm{T}}=(3 / 2)\left(1-j^{2}\right)$. It is also noted that only in regions 3,6 , and 9 there exist two stable equilibrium positions. The hybrid curves corresponding to the boundary of these bi-stability regions are signified by the thick lines in Fig. 2. This boundary comprises parts of $e_{2}-e_{\mathrm{T}}$ and $e_{1}-e_{\mathrm{T}}$ curves, and will be called $e_{1}^{\prime}-e_{\mathrm{T}}$ in the later sections.

For a specified rise parameter $h$ we can draw a horizontal line for $e=0$ and a vertical line for $q_{1}=0$, whose intersection is denoted by a black dot in Fig. 2. These points signify the unloaded and unstretched arch for a particular $h$. Several different $h$ 's are chosen in Fig. 2 to demonstrate the quasi-static behavior of the arch. Point (a) at $\left(q_{1}-h, e-e_{\mathrm{T}}\right)=(0,1)$ is for $h=0$, an initially straight rod. For the conventional case 


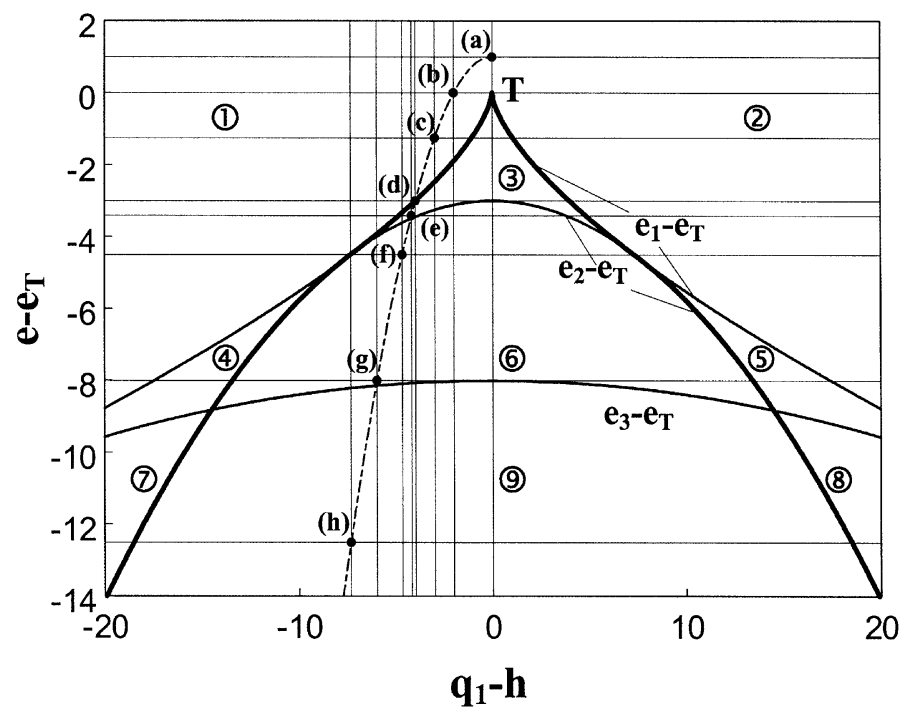

Fig. 2. Bifurcation set with $q_{1}-h$ and $e-e_{\mathrm{T}}$ as control parameters. The small dots represent the parameters $q_{1}=0$ and $e=0$ for various $h$.

Table 1

Equilibrium configurations and stability of various regions in Fig. 2

\begin{tabular}{ll}
\hline Region & Equilibrium configurations \\
\hline 1 & $\mathbf{P}_{\mathbf{0}}$ \\
2 & $\mathbf{P}_{\mathbf{1}}^{-}$ \\
3 & $\mathbf{P}_{\mathbf{0}}, P_{1}^{+}, \mathbf{P}_{\mathbf{1}}^{-}$ \\
4 & $\mathbf{P}_{\mathbf{0}}, P_{1}^{+}, P_{1}^{-}$ \\
5 & $P_{0}, P_{1}^{+}, \mathbf{P}_{\mathbf{1}}^{-}$ \\
6 & $\mathbf{P}_{\mathbf{0}}, P_{1}^{+}, \mathbf{P}_{\mathbf{1}}^{-}, P_{12}^{+}, P_{12}^{-}$ \\
7 & $\mathbf{P}_{\mathbf{0}}, P_{1}^{+}, P_{1}^{-}, P_{13}^{+}, P_{13}^{-}$ \\
8 & $P_{0}, P_{1}^{+}, \mathbf{P}_{\mathbf{1}}^{-}, P_{13}^{+}, P_{13}^{-}$ \\
9 & $\mathbf{P}_{\mathbf{0}}, P_{1}^{+}, \mathbf{P}_{\mathbf{1}}^{-}, P_{12}^{+}, P_{12}^{-}, P_{13}^{+}, P_{13}^{-}$ \\
\hline
\end{tabular}

when $e=0$ (along the horizontal line passing through this point) there exists only $P_{0}$ or $P_{1}^{-}$solution for $q_{1}<0$ or $q_{1}>0$, respectively. On the other hand when $q_{1}=0$ (along the vertical line) and $e$ is decreased to $-4<e<-1$, there exist three solutions $P_{0}, P_{1}^{+}$, and $P_{1}^{-}$. It is noted that when $e=-1$ the axial thrust $p=1$ corresponds to the first buckling load. When $e$ is decreased to the range $-9<e<-4$, there exist five solutions, i.e., $P_{0}, P_{1}^{+}, P_{1}^{-}, P_{12}^{+}$, and $P_{12}^{-}$. $e=-4$ corresponds to the second buckling load $p=4$.

Point (b) at $(-2,0)$ is for $h=2$. The scenarios along the $e=0$ line and the $q_{1}=0$ line are similar to the case at Point (a), except that the tip T is right on the $e=0$ line. As a consequence for an arch with $h<2$, there exists only one solution along the $e=0$ line, and no snap-through is possible (Hoff and Bruce, 1954). It is also noted that as $e$ decreases along the $q_{1}=0$ line across the $e_{1}-e_{\mathrm{T}}$ curve, the number of possible solutions increases from 1 to 3 . On the $e_{1}-e_{\mathrm{T}}$ curve the solutions $P_{1}^{+}$and $P_{1}^{-}$coincide and the degenerate root can be found as

$$
\alpha_{1}=-h-\left[2\left(h-q_{1}\right)\right]^{1 / 3} .
$$


Point (c) is for $h=3$, in which the tip $\mathrm{T}$ is above the $e=0$ line and to the right of the $q_{1}=0$ line. In this case there exist $q_{11}$ and $q_{12}$ on the $e=0$ line such that there are three solutions $P_{0}, P_{1}^{+}$, and $P_{1}^{-}$when $q_{1}$ is in the range $q_{11}<q_{1}<q_{12}$, where

$$
\begin{aligned}
& q_{11}=h-\frac{\left(h^{2}-4\right)^{3 / 2}}{6 \sqrt{3}}, \\
& q_{12}=h+\frac{\left(h^{2}-4\right)^{3 / 2}}{6 \sqrt{3}} .
\end{aligned}
$$

When $q_{1}<q_{11}$ the only equilibrium configuration is $P_{0}$. When $q_{1}>q_{12}$ the only equilibrium configuration is $P_{1}^{-}$. If $q_{1}$ is increased from 0 quasi-statically, the arch will be snapped from $P_{0}$ to $P_{1}^{-}$when $q_{1}=q_{12}$. On the other hand when $q_{1}$ is decreased from beyond $q_{12}$ down to $q_{11}$, then the arch will be snapped from $P_{1}^{-}$to $P_{0}$.

Point (d) for $h=4$ is right on the left branch of the $e_{1}-e_{\mathrm{T}}$ curve. Also the $e_{2}-e_{\mathrm{T}}$ curve touches the $e=0$ line. Therefore, for the unloaded $\left(q_{1}=0\right)$ and unstretched $(e=0)$ arch multiple equilibrium positions are possible only when $h>4$. In addition, $P_{12}^{+}$, and $P_{12}^{-}$exist along the $e=0$ line only when $h>4$.

Points (e), (f), and (g) are for $h=4.2, \sqrt{22}$, and 6 , and are above, right on, and below the horizontal line passing through the touching points of $e_{1}-e_{\mathrm{T}}$ and $e_{2}-e_{\mathrm{T}}$ curves, respectively. The $e_{2}-e_{\mathrm{T}}$ curves intersect the $e=0$ line in each of these three cases at $q_{21}$ and $q_{22}$, where

$$
\begin{aligned}
& q_{21}=h-3 \sqrt{h^{2}-16}, \\
& q_{22}=h+3 \sqrt{h^{2}-16} .
\end{aligned}
$$

For $h=4.2$ when the load increases along the $e=0$ line static snap-through will occur when $q_{1}$ reaches $q_{12}$. On the other hand when $h=6$, static snap-through will occur when $q_{1}$ reaches $q_{22}$. In the case when $h=\sqrt{22}, q_{11}=q_{21}$ and $q_{12}=q_{22}$. Point (h) is for $h=3 \sqrt{6}$, which is on the vertical line passing through the left touching point of the $e_{1}-e_{\mathrm{T}}$ and $e_{2}-e_{\mathrm{T}}$ curves. All the black dots in Fig. 2 are on a parabola, indicated by the dashed line

$$
q_{1}-h=1-\frac{1}{4}\left(e-e_{\mathrm{T}}\right)^{2}
$$

In Fig. 3 we plot the relation between $\alpha_{1}$ and $e$ for $h=6$ (point (g) in Fig. 2). The loads $q_{1}$ 's in Fig. 3(a), (b), (c), and (d) are $1-3 \sqrt{6}, 11-3 \sqrt{6}, 11+3 \sqrt{6}$, and $1+3 \sqrt{6}$, respectively. These $q_{1}$ 's satisfy the conditions (a) $q_{1}<h,\left|q_{1}-h\right|>3 \sqrt{6}$, (b) $q_{1}<h,\left|q_{1}-h\right|<3 \sqrt{6}$, (c) $q_{1}>h,\left|q_{1}-h\right|>3 \sqrt{6}$, (d) $q_{1}>h$, $\left|q_{1}-h\right|<3 \sqrt{6}$. Both $e_{1}$ and $e_{2}$ are positive in Fig. 3. There are five equilibrium configurations when $e=0$, among them either $P_{0}$ or $P_{1}^{-}$can be the initial position before any end motion takes place, as denoted by small black dots. The solid and dashed lines represent the stable and unstable configurations, respectively. For the cases in Fig. 3(a) and (b), if the arch is in $P_{0}$ position when $e=0$ and is stretched or compressed quasi-statically, the arch will always stay in $P_{0}$ position. On the other hand, if the arch is in $P_{1}^{-}$position and stretched quasi-statically, then the arch will snap to $P_{0}$ position either via a sub-critical pitchfork bifurcation when $e$ reaches $e_{2}$ as in Fig. 3(a), or via a saddle-node bifurcation at $e_{1}$ as shown in Fig. 3(b). In Fig. 3(c) and (d), if the arch is in $P_{1}^{-}$position when $e=0$ and is stretched or compressed quasi-statically, the arch will always stay in $P_{1}^{-}$position. On the other hand, if the arch is in $P_{0}$ position and stretched quasi-statically, then the arch will snap to $P_{1}^{-}$position either via a sub-critical pitchfork bifurcation when $e$ reaches $e_{2}$ as shown in Fig. 3(c), or via a saddle-node bifurcation at $e_{1}$ as shown in Fig. 3(d). 

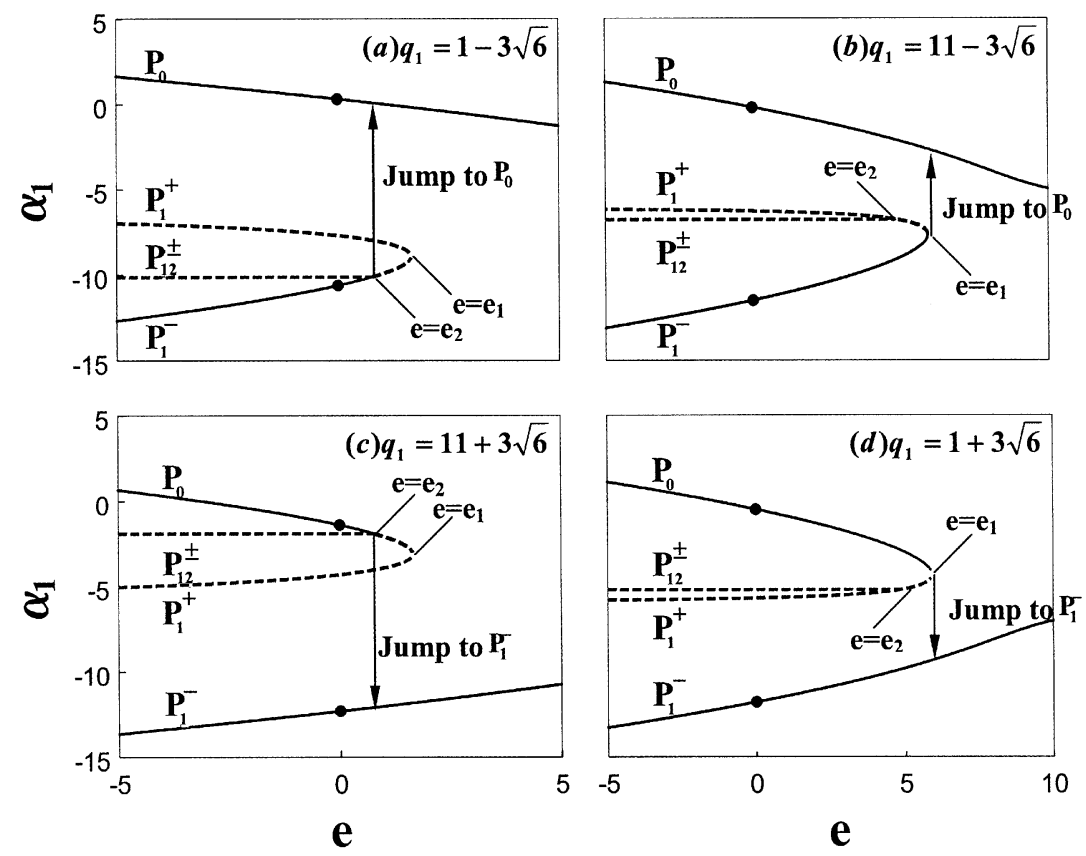

Fig. 3. Equilibrium configurations for $h=6$ with various $q_{1}$.

\section{Dynamic snap-through criteria}

In the case when the end speed is not negligible dynamic snap-through may occur. For dynamic snapthrough to occur there must exist at least two stable equilibrium configurations when the motion of the arch end stops. While it is in general difficult to determine the necessary and sufficient condition for dynamic snap-through to occur, we can establish the sufficient conditions against dynamic snap-through in terms of dimensionless strain energy $U$ of the equilibrium configurations and the total energy $H$ gained by the arch at the instant when the arch end stops, where

$$
\begin{aligned}
& H=2(G+e)^{2}+\sum_{n=1}^{\infty}\left[\dot{\alpha}_{n}^{2}+n^{4} \alpha_{n}^{2}\right]+2 q_{1}\left(\alpha_{1}+h\right), \\
& U=2(G+e)^{2}+\sum_{n=1}^{\infty} n^{4} \alpha_{n}^{2}+2 q_{1}\left(\alpha_{1}+h\right) .
\end{aligned}
$$

The physical total energy $H^{*}$ and strain energy $U^{*}$ are related to $H$ and $U$ by

$$
H^{*}=\frac{\pi^{4} E I^{2} H}{4 A L^{3}}, \quad U^{*}=\frac{\pi^{4} E I^{2} U}{4 A L^{3}} .
$$

The basic idea is that if the total energy gained by the arch during the prescribed end motion is smaller than the minimum energy barrier lying between the nearest stable equilibrium position and the distant stable one, then the arch has no chance to snap dynamically. The energy barrier can be proved to be the strain energy of either the unstable configuration $P_{1}^{+}$or $P_{12}^{ \pm}$, depending on the parameters $q_{1}, h$ and $e$ (Lin, 2002). If we assume that the arch is in position $P_{0}$ before any end motion, then the sufficient conditions against dynamic snap-through from $P_{0}$ to $P_{1}^{-}$can be stated in the following: 
Case (1) $\left|q_{1}-h\right|<3 \sqrt{6}$ : If $e_{2}<e<e_{1}$, then the sufficient condition against snap-through is $\alpha_{1}\left(\tau_{\mathrm{s}}\right)>\alpha_{1}\left(P_{1}^{+}\right)$ and $H\left(\tau_{\mathrm{s}}\right)<U\left(P_{1}^{+}\right)$, where $\tau_{\mathrm{s}}$ is the time when the arch end stops. If $e<e_{2}$, then the sufficient condition against snap-through is $\alpha_{1}\left(\tau_{\mathrm{s}}\right)>\alpha_{1}\left(P_{12}^{ \pm}\right)$and $H\left(\tau_{\mathrm{s}}\right)<U\left(P_{12}^{ \pm}\right)$.

Case (2) $\left|q_{1}-h\right|>3 \sqrt{6}$ : If $e<e_{2}$ then the sufficient condition against snap-through is $\alpha_{1}\left(\tau_{\mathrm{s}}\right)>\alpha_{1}\left(P_{12}^{ \pm}\right)$and $H\left(\tau_{\mathrm{s}}\right)<U\left(P_{12}^{ \pm}\right)$.

In the case when the arch is in position $P_{1}^{-}$before any end motion, then the above statements are slightly modified by changing $\alpha_{1}\left(\tau_{\mathrm{s}}\right)>\alpha_{1}\left(P_{12}^{ \pm}\right)$and $\alpha_{1}\left(\tau_{\mathrm{s}}\right)>\alpha_{1}\left(P_{1}^{+}\right)$to $\alpha_{1}\left(\tau_{\mathrm{s}}\right)<\alpha_{1}\left(P_{12}^{ \pm}\right)$and $\alpha_{1}\left(\tau_{\mathrm{s}}\right)<\alpha_{1}\left(P_{1}^{+}\right)$, respectively. These two statements give the sufficient conditions against dynamic snap-through if the deflection $\alpha_{1}\left(\tau_{\mathrm{s}}\right)$ and total energy $H\left(\tau_{\mathrm{s}}\right)$ are known, which require direct integration of the equations of motion. Instead of calculating $H\left(\tau_{\mathrm{s}}\right)$, we can estimate the upper bound of total energy gained by the arch during the end motion. It can be proved that the total energy gained by the arch when the end is moved with infinitely large speed is an upper bound of the total energy gained by the arch stretched with finite speed (Lin, 2002). The total energy corresponding to $c \rightarrow \infty$ is denoted by $H_{\infty}$, and can be calculated as

$$
H_{\infty}=\left(\alpha_{1}^{0}\right)^{2}+2\left[\frac{\left(\alpha_{1}^{0}\right)^{2}}{4}+\frac{h \alpha_{1}^{0}}{2}+e\right]^{2}+2 q_{1}\left(h+\alpha_{1}^{0}\right),
$$

where $\alpha_{1}^{0}$ is the deflection of the arch under lateral load $q_{1}$ before any end motion. In the case when $q_{1}=0$, then $\alpha_{1}^{0}=0$ and $H_{\infty}$ is reduced to $2 e^{2}$. Finally we can establish a simpler, although more conservative, sufficient condition against dynamic snap-through by replacing $H\left(\tau_{\mathrm{s}}\right)$ in the snap-through criteria mentioned above by $H_{\infty}$. It can be shown that there exists a critical distance $e_{\mathrm{cr}}$, such that

$$
H_{\infty}\left(e_{\mathrm{cr}}\right)=U\left(P_{1}^{+}\right)
$$

when $\left|q_{1}-h\right|<3 \sqrt{6}$ and $e_{2} \leqslant e<e_{1}$. In all other cases,

$$
H_{\infty}\left(e_{\mathrm{cr}}\right)=U\left(P_{12}^{ \pm}\right) \text {. }
$$

$H_{\infty}\left(e_{\mathrm{cr}}\right)$ in Eqs. (34) and (35) represents the total energy gained by the arch when its end is moved a distance $e_{\mathrm{cr}}$ with infinitely large speed. It is noted that we allow $e_{\mathrm{cr}}$ to be either positive or negative.

In Fig. 4 we plot the $e_{\text {cr }}$ curves with dashed lines on the $q_{1}-e$ plane. The $q_{1}=0$ line, $e=0$ line, and the $e_{1}$ and $e_{2}$ curves are also presented for reference. For a specified $q_{1}$ if we move the arch end from $e=0$ into the cross-hatched area then there is a possibility of dynamic snap-through. The cross-hatched area may be called the "dangerous zone." If the arch is in $P_{0}$ position when $e=0$, then the lines in the cross-hatched area are of positive slope. On the other hand if the arch is in $P_{1}^{-}$position when $e=0$, then the lines in the crosshatched area are of negative slope. In Fig. 4(a) for $h=2$ no dynamic snap-through is possible during stretching process because there is only one stable equilibrium position when $e>0$ for $h \leqslant 2$. However, dynamic snap-through is possible during the compressing process. In Fig. 4(b) for $h=4$ the sharp corners of the two dangerous zones during stretching process are located at $\left(q_{1}, e\right)=(0,0)$ and $(8,0)$. Let's take $q_{1}=6$ as an example. If the arch is in $P_{0}$ position when $e=0$, then there is a risk of dynamic snap-through if the arch is stretched a distance in the range from 0.75 to 1.11 , or compressed a distance over 5.31. On the other hand, if the arch is in $P_{1}^{-}$position when $e=0$, then the arch is moved to the dangerous zone when it is compressed a distance over 8.96. There is no risk of snap-through from stretching in this case. In Fig. 4(c) for $h=\sqrt{22}$ the sharp corners of the dangerous zones during both stretching and compressing processes coincide with the two touching points of the $e_{1}$ and $e_{2}$ curves. In Fig. 4(d) for $h=3 \sqrt{6}$ the sharp corners of the dangerous zones during both stretching and compressing processes coincide and are on the $e_{2}$-curves. In addition, the other corners of the dangerous zones during stretching process coincide with the two touching points of the $e_{1}$ and $e_{2}$ curves. Fig. 4(d) indicates that for an unloaded arch in the $P_{0}$ position dynamic snapthrough during stretching is possible only when $h>3 \sqrt{6}$. 

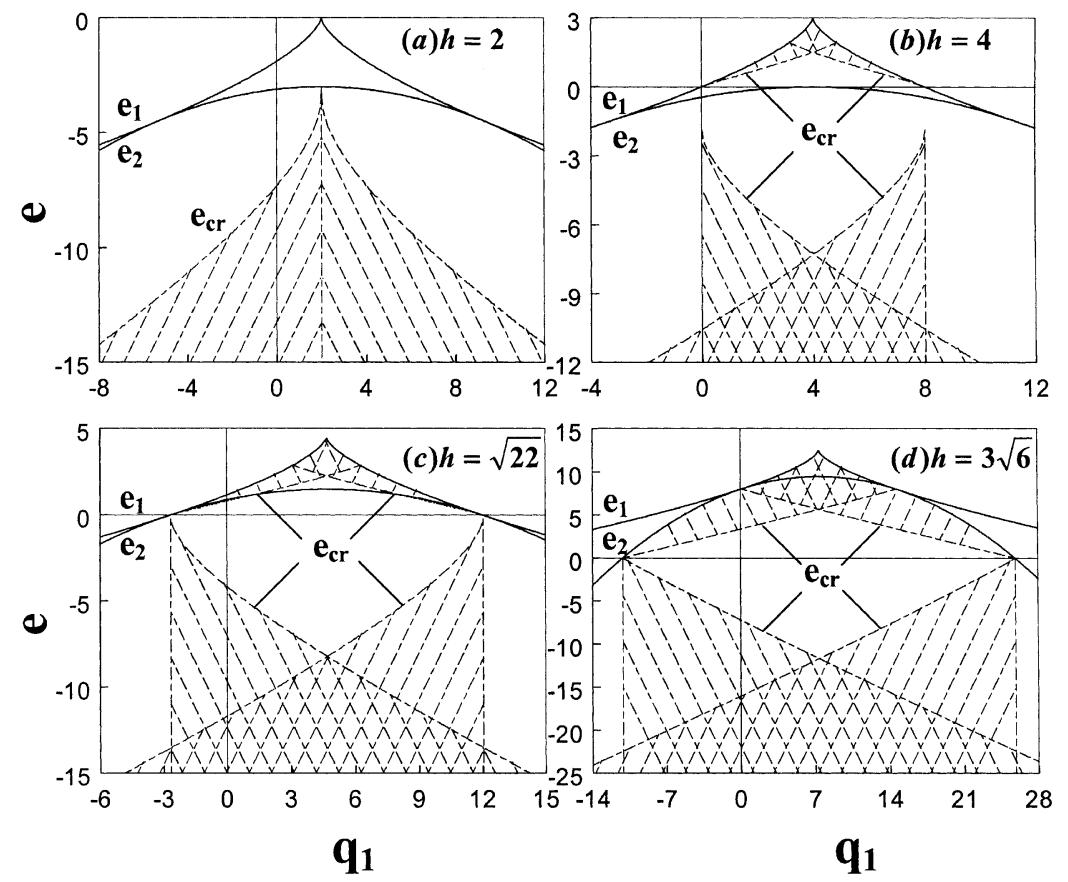

Fig. 4. The dangerous zones for (a) $h=2$, (b) $h=4$, (c) $h=\sqrt{22}$, and (d) $h=3 \sqrt{6}$.

\section{Dynamic snap-through during compression}

The above analysis predicts that the loaded arch can snap dynamically when the loaded arch is either stretched or compressed. While it is easier to visualize the snapping of a stretched arch, it is not obvious how a loaded arch snaps when it is compressed dynamically. In Fig. 4 we demonstrate that the arch may indeed undergo dynamic snap-through when it is compressed. The reason for this seemingly unreasonable result is that we consider the minimum energy barrier in establishing the sufficient condition against dynamic snap-through, which turns out to be the strain energy of the positions $P_{12}^{ \pm}$if these positions exist when the arch end stops. However, in the case when the arch is compressed with ideal initial conditions (16) and (17) with $\alpha_{n}^{0}=0 n \neq 1$, then the only possible non-zero coordinate is $\alpha_{1}$. Therefore, the arch has no chance to pass the saddle point $P_{12}^{ \pm}$, which involves both $\alpha_{1}$ and $\alpha_{2}$. In order to reach the other stable equilibrium position $P_{1}^{-}, H_{\infty}$ has to surpass the energy barrier at $P_{1}^{+}$. It can be shown that $H_{\infty}<U\left(P_{1}^{+}\right)$in the compressing process. Therefore, an arch with ideal initial conditions will never undergo snap-through when it is compressed dynamically.

The sufficient conditions presented in Fig. 4 are conservative in the sense that it implicitly takes into account various minor imperfections, such as the non-ideal initial conditions or the imperfect initial shape. Due to these imperfections the coordinates other than $\alpha_{1}$, such as $\alpha_{2}$, may be aroused. It is then possible for the arch to pass through the saddle point $P_{12}^{ \pm}$. Therefore, it will be safer to stick to the true minimum energy barrier $U\left(P_{12}^{ \pm}\right)$in asserting the sufficient conditions.

To demonstrate how non-ideal initial conditions may cause dynamic snap-through when the arch is compressed, we consider the case when $q_{1}=7, h=5, e=-10$, and $c=-40$. The arch is in $P_{0}$ position before the prescribed end motion. Initial conditions are the same as (16) and (17), except that we change $\alpha_{2}(0)$ from 0 to 0.001 . In the numerical simulation we add damping terms $\mu \dot{\alpha}_{1}$ and $\mu \dot{\alpha}_{2}$ in Eqs. (10) and (11), where we choose $\mu=0.01$. Fig. 5 shows the deformations $\alpha_{1}$ and $\alpha_{2}$ as functions of $\tau$. We observe that in the 

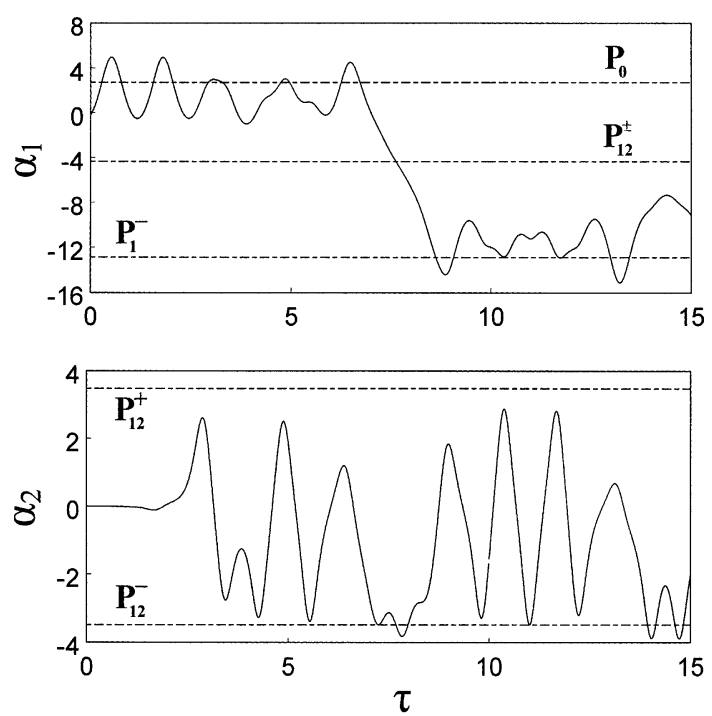

Fig. 5. Response history during compression for $q_{1}=7, h=5, e=-10, c=-40$, and $\mu=0.01$. Initial conditions are the same as (16) and (17), except that $\alpha_{2}(0)$ is changed from 0 to 0.001 .

early stage $\tau<2, \alpha_{1}$ is mostly positive as we may expect and $\alpha_{2}$ remains small. However, after $\tau>2$ the amplitude of $\alpha_{2}$-oscillation grows significantly and $\alpha_{1}$ shoots to the negative territory at $\tau=7$ and remains negative thereafter. The coordinates of equilibrium positions $P_{0}, P_{1}^{-}$and $P_{12}^{ \pm}$corresponding to $e=-10$ are shown as dashed lines for reference. In Fig. 6 we use the thick line to trace the trajectory of the deformation history in the $\alpha_{1}-\alpha_{2}$ space. Also shown is the strain energy contour and the locations of various equilibrium positions corresponding to $e=-10$. The trajectory starts near the valley $P_{0}$ and slides back and forth several times along an almost horizontal line (recall the small $\alpha_{2}$ in the early stage). Since the system is unable to climb to the hilltop at $P_{1}^{+}$, it naturally swirls up the wall surrounding $P_{0}$ and finds an easier route

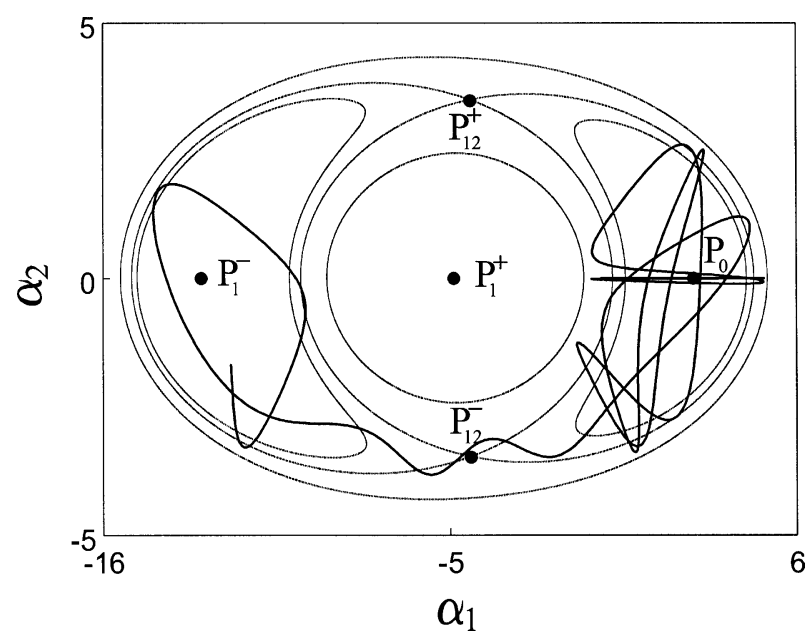

Fig. 6. Trajectory of the response in Fig. 5 in the $\alpha_{1}-\alpha_{2}$ space. The equilibrium positions and strain energy contour for $e=-10$ are also shown. 
passing the saddle point $P_{12}^{-}$and reaches the other valley $P_{1}^{-}$. Consequently dynamic snap-through occurs when the arch is under compression with non-ideal initial conditions.

\section{Effects of $q_{2}$ on equilibrium positions}

\subsection{Two-mode solution}

In this section we consider the effects of the second harmonic component $q_{2}$ of the distributed load. Apparently there is no more one-mode solution when both $q_{1}$ and $q_{2}$ are present. Instead, the $\alpha_{1}$ and $\alpha_{2}$ of a two-mode solution satisfy the following equations:

$$
\begin{aligned}
& \alpha_{1}+(G+e)\left(h+\alpha_{1}\right)+q_{1}=0, \\
& 16 \alpha_{2}+4(G+e) \alpha_{2}+q_{2}=0,
\end{aligned}
$$

where

$$
G=\frac{1}{4}\left(\alpha_{1}^{2}+4 \alpha_{2}^{2}\right)+\frac{h}{2} \alpha_{1}
$$

If the notation $\alpha_{1}\left(P_{12}^{ \pm}\right)$is used to represent the two-mode solution when $q_{2}=0$, which is given in Eq. (21), then after eliminating $(G+e)$ in Eqs. (36) and (37) $\alpha_{2}$ and $\alpha_{1}$ can be related by

$$
\alpha_{2}=-\frac{\left(\alpha_{1}+h\right) q_{2}}{12\left[\alpha_{1}-\alpha_{1}\left(P_{12}^{ \pm}\right)\right]} .
$$

After substituting Eq. (39) into Eq. (36), one can derive the equation for $\alpha_{1}$

$$
36\left[\alpha_{1}-\alpha_{1}\left(P_{12}^{ \pm}\right)\right]^{2}\left[\alpha_{1}^{3}+3 \alpha_{1}^{2} h+2 \alpha_{1}\left(2+2 e+h^{2}\right)+4\left(h e+q_{1}\right)\right]+\left(\alpha_{1}+h\right)^{3} q_{2}^{2}=0 .
$$

The first bracket represents the original two-mode solution and the second bracket represents the original one-mode solution when $q_{2}=0$. Eqs. (39) and (40) can be used to study the effects of $q_{2}$ on the equilibrium configurations. As expected the sign of $q_{2}$ has no effect on the root locus of $\alpha_{1}$. There are a total of five poles and five zeros in Eq. (40), among them $\alpha_{1}=-h$ is a triple zero.

Case (1) $q_{1}<h$ :

For $e>e_{1}$ there are two possible scenarios for the root loci of $\alpha_{1}$ and $\alpha_{2}$, as shown in Fig. 7(a) and (b). In each scenario there is only one real equilibrium $P_{0}$ when $q_{2}=0$. In Fig. 7(a) as $q_{2}$ increases from zero to infinity, $\alpha_{1}\left(P_{0}\right)$ approaches the zero $\alpha_{1}=-h$ while the corresponding $\alpha_{2}$ approaches $-\infty$ along the real axis. The complex conjugate poles $\alpha_{1}\left(P_{1}^{ \pm}\right)$approach infinity while the corresponding $\alpha_{2}$ break away from the real axis at the origin and approach infinity. When $q_{2}=0 \alpha_{1}\left(P_{12}^{ \pm}\right)$are on the real axis but the corresponding $\alpha_{2}$ are complex conjugate pairs. As $q_{2}$ increases from zero $\alpha_{1}\left(P_{12}^{ \pm}\right)$break away from the real axis and approach the zero $\alpha_{1}=-h$, while the corresponding $\alpha_{2}$ remain complex and approach infinity. The situation in Fig. 7(b) is similar to that in Fig. 7(a) except that in Fig. 7(b) the poles $\alpha_{1}\left(P_{12}^{ \pm}\right)$approach infinity and $\alpha_{1}\left(P_{1}^{ \pm}\right)$ approach the zero $\alpha_{1}=-h$. Therefore, in either Fig. 7(a) or (b) the number of real equilibrium position remains to be one. In addition, $q_{2}$ tends to suppress the deformed configuration $\left(h+\alpha_{1}\right) \sin \xi$ contributed from $q_{1}$. In the limiting case when $q_{2} \rightarrow \infty$ the arch is deformed to the form $\alpha_{2} \sin 2 \xi$.

There are also two possible scenarios for the root loci of $\alpha_{1}$ and $\alpha_{2}$ for $e_{1}>e>e_{2}$, in which there are three equilibrium positions $P_{0}, P_{1}^{+}$, and $P_{1}^{-}$when $q_{2}=0$ as shown in Fig. 7(c) and (d). Careful observation reveals that $q_{2}$ tends to reduce the number of equilibrium positions from three to one. Fig. 7(e) shows the 
(a)

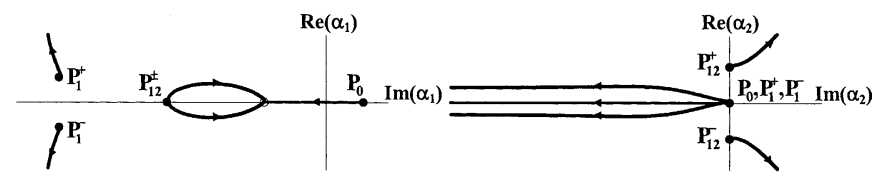

(b)

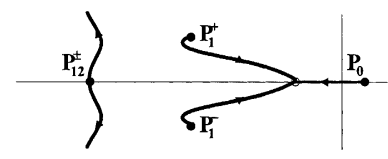

(c)

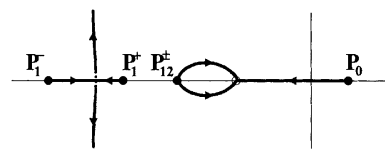

(d)

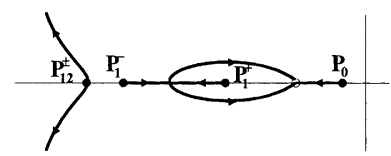

(e)

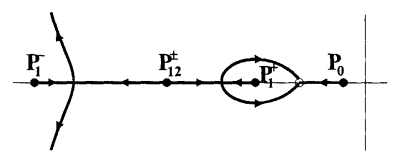

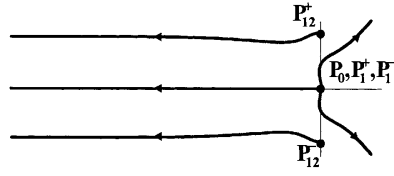
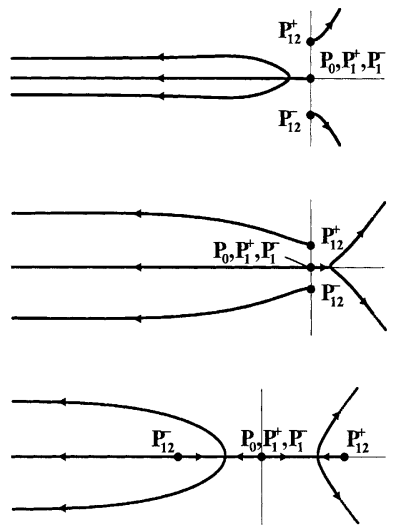

Fig. 7. Root loci for $\alpha_{1}$ and $\alpha_{2}$ as $q_{2}$ increases from 0 to $\infty$ when $q_{1}<h$. (a, b) For $e>e_{1}$, (c, d) for $e_{1}>e>e_{2}$ and (e) for $e<e_{2}$.

case when $e<e_{2}$, in which there are five equilibrium positions when $q_{2}=0$. As $q_{2}$ increases from 0 , the number of equilibrium positions reduces from five to three and finally to one as $q_{2}$ increases.

Case (2) $q_{1}>h$ :

The root loci in this case are similar to those in Fig. 7. The main difference between Case (2) and Case (1) is the exchange of the roles played by $P_{0}$ and $P_{1}^{-}$. The conclusion remains the same that $q_{2}$ tends to reduce the number of equilibrium positions to one.

The effects of $q_{2}$ on the root loci discussed above can also be demonstrated in the $\alpha_{i}-e$ curves as shown in Fig. 8, in which we choose $h=6, q_{1}=1-3 \sqrt{6}$, and $q_{2}=10$. The $\alpha_{1}$-curves for $q_{2}=0$ have been presented in Fig. 3(a) and are replotted in Fig. 8 as thin lines for reference. It is seen that the original sub-critical pitchfork bifurcation at $e=e_{2}$ is destroyed and two separate branches are created. The two new saddlenode bifurcation points are at $e=e_{1}^{\prime}$ and $e_{2}^{\prime}$. It is noted that $e_{1}^{\prime}$ is defined in such a way that two stable equilibrium positions coexist when $e<e_{1}^{\prime}$. It is reminded that the $e_{1}^{\prime}$ for $q_{2}=0$ corresponds to the thick hybrid curve in Fig. 2. The new $\alpha_{1}$-curve for $P_{0}$ solution is slightly lower than and is indistinguishable from the original one with the chosen parameters. As in Fig. 3 the solid and dashed lines represent stable and unstable solutions, respectively.

Case (3) $q_{1}=h$ :

This is a limiting case of both Cases (1) and (2), but there are some interesting phenomena worth mentioning separately. First of all Eqs. (39) and (40) for the root loci fail in this case. Instead, we rewrite Eq. (36) to the form

$$
\left(\alpha_{1}+h\right)(G+e+1)=0 .
$$



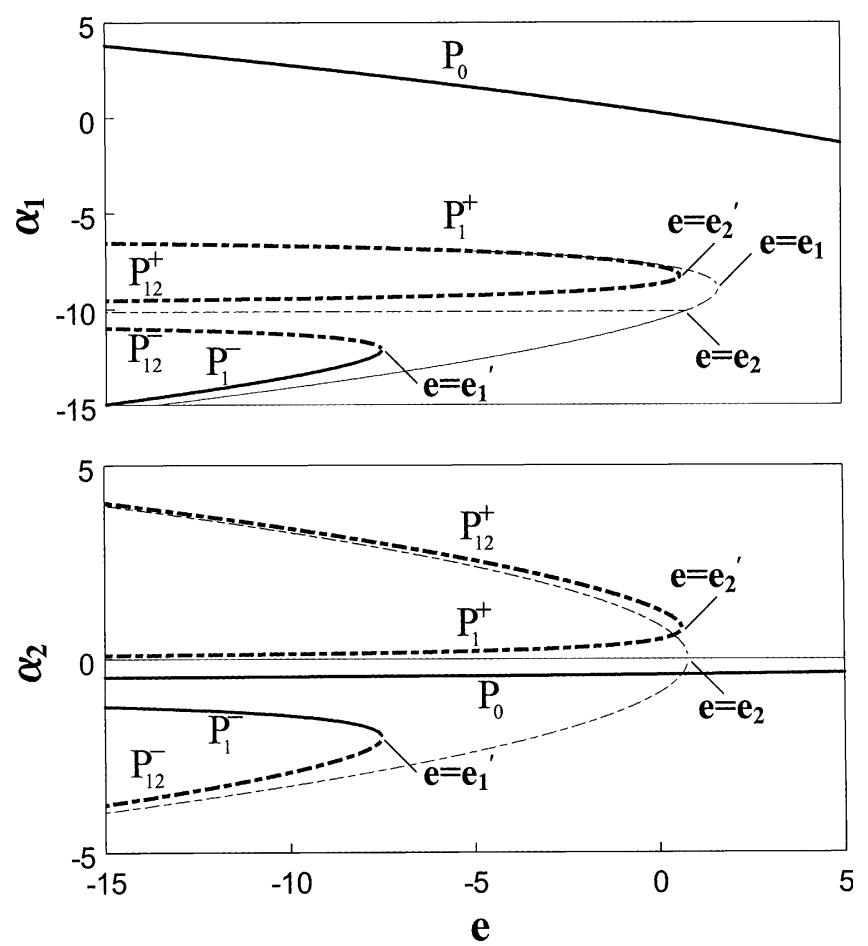

Fig. 8. Equilibrium configurations for $h=6$ and $q_{1}=1-3 \sqrt{6}$. The thin lines are for $q_{2}=0$ and the thick lines are for $q_{2}=10$.

For solution

$$
\alpha_{1}=-h,
$$

the corresponding $\alpha_{2}$ satisfies the equation

$$
4 \alpha_{2}\left(\alpha_{2}^{2}+e-e_{2}\right)+q_{2}=0 .
$$

On the other hand, for solution $G+e=-1$ the corresponding $\alpha_{2}$ satisfies the equation

$$
\alpha_{2}=-\frac{q_{2}}{12} \text {. }
$$

After substituting Eq. (44) back to $G+e=-1$ the corresponding $\alpha_{1}$ are

$$
\alpha_{1}=-h \pm\left[h^{2}-4\left(\frac{q_{2}^{2}}{144}+e+1\right)\right]^{\frac{1}{2}} \text {. }
$$

Eqs. (42)-(45) can be used to plot the root loci for $\alpha_{1}$ and $\alpha_{2}$ in this case. Alternatively, one can also use Fig. 7(a), (c), and (e) and let $q_{1}$ approach $h$ to explain the root behaviors in this limiting case.

Fig. 9(a) shows the case for $e>e_{1}$. When $q_{2}=0$ the three $\alpha_{1}$ 's for $P_{0}$ and $P_{12}^{ \pm}$coalesce with the triple zero $\alpha_{1}=-h$ in Fig. 7(a) and the three $\alpha_{2}$ 's for $P_{0}$ and $P_{1}^{ \pm}$are at the origin. Therefore there exists only one real equilibrium at $\left(\alpha_{1}, \alpha_{2}\right)=(-h, 0)$. As $q_{2}$ increases from zero, the three poles at $\alpha_{1}=-h$ do not move, while the three poles at $\alpha_{2}=0$ approach $-\infty$ along the real axis together. Consequently, the number of 
(a)

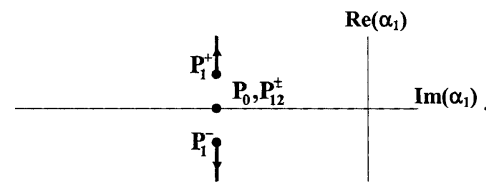

(b)

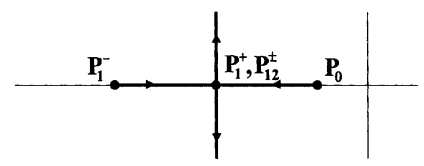

(c)

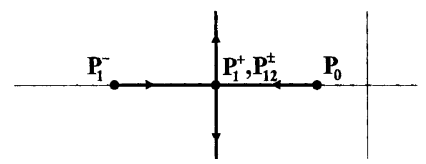

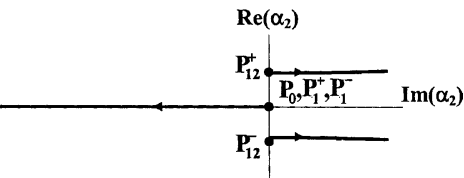
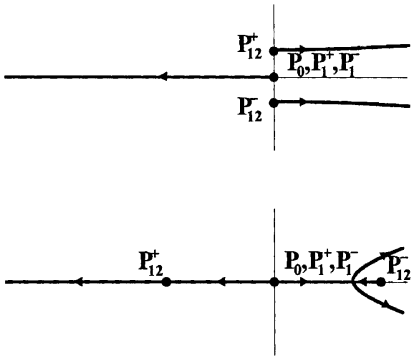

Fig. 9. Root loci for $\alpha_{1}$ and $\alpha_{2}$ as $q_{2}$ increases from 0 to $\infty$ when $q_{1}=h$. (a) For $e>e_{1}$, (b) for $e_{1}>e>e_{2}$ and (c) for $e<e_{2}$.

equilibrium position remains to be one. Fig. 9(b) and (c) are for the cases $e_{1}>e>e_{2}$, and $e<e_{2}$, respectively.

Fig. 10 shows the $\alpha_{i}-e$ curves when $q_{1}=h=6$. The thin lines and the thick lines represent the solutions for $q_{2}=0$ and $q_{2}=10$, respectively. For $q_{2}=0$ the arch undergoes super-critical pitchfork bifurcation at
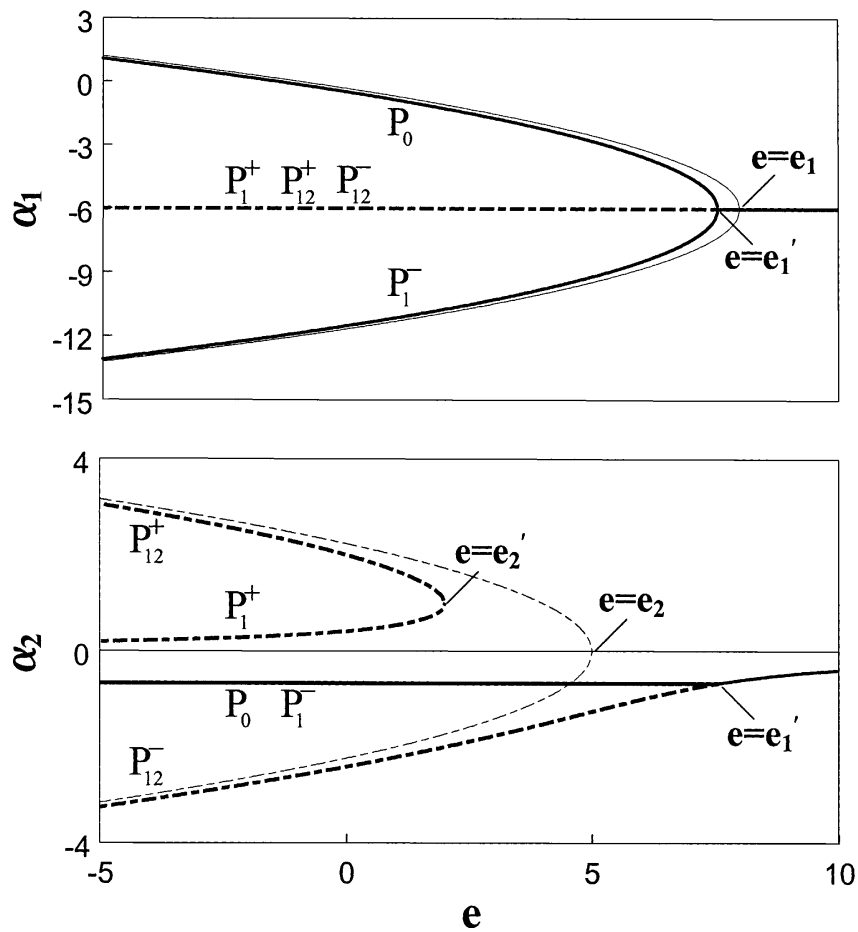

Fig. 10. Equilibrium configurations for $q_{1}=h=6$. The thin lines are for $q_{2}=0$ and the thick lines are for $q_{2}=10$. 
both $e=e_{1}$ and $e_{2}$. The numbers of equilibrium positions are 1, 3, and 5 when $e>e_{1}, e_{1}>e>e_{2}$, and $e<e_{2}$, respectively. When $q_{2} \neq 0$, the bifurcation points are shifted leftward to $e_{1}^{\prime}$ and $e_{2}^{\prime}$. It is noted that the arch undergoes a super-critical pitchfork bifurcation at $e=e_{1}^{\prime}$. This is different from the saddle-node bifurcation in Fig. 8 when $q_{1} \neq h$.

\subsection{Three-mode solution}

When both $q_{1}$ and $q_{2}$ are non-zero three-mode solutions are possible, with $\alpha_{1}$ being given by Eq. (21) and $\alpha_{2}$ and $\alpha_{j}$ being

$$
\begin{aligned}
& \alpha_{2}=\frac{q_{2}}{4\left(j^{2}-4\right)}, \\
& \alpha_{j}= \pm \frac{2}{j} \sqrt{e_{j}^{\prime}-e}, \quad j>2,
\end{aligned}
$$

where

$$
e_{j}^{\prime}=e_{j}-\frac{q_{2}^{2}}{16\left(j^{2}-4\right)^{2}} .
$$

In the case when $q_{2}$ approaches zero, the three-mode solutions are reduced to the original two-mode solutions with $\alpha_{j}$ in Eq. (47) approaching the one in Eq. (22). All these three-mode solutions are unstable. In addition, the existence of these three-mode solutions will not affect the stability of the two-mode solutions as shown in Figs. 8 and 10. The effects of other $q_{j}$ with $j>2$ can be studied in a similar manner.

\section{Effects of $q_{2}$ on dynamic stability}

In order to study the effects of $q_{2}$ on the dangerous zone in Fig. 4, we have to consider three factors. First of all for all other parameters being unchanged $q_{2}$ tends to reduce the number of stable equilibrium positions from 2 to 1 , as explained in the last section. Secondly, when $q_{2}$ is non-zero the strain energy of positions $P_{12}^{+}$and $P_{12}^{-}$are no longer the same,

$$
U\left(P_{12}^{ \pm}\right)=2\left(\frac{h \alpha_{1}}{2}+\frac{\alpha_{1}^{2}}{4}+\alpha_{2}^{2}+e\right)^{2}+\alpha_{1}^{2}+16 \alpha_{2}^{2}+2 q_{1}\left(\alpha_{1}+h\right)+2 q_{2} \alpha_{2},
$$

$\alpha_{1}$ and $\alpha_{2}$ in Eq. (49) are coordinates of the equilibrium positions $P_{12}^{ \pm}$of the laterally loaded arch under end displacement $e$, and are dependent upon $q_{2}$. Further calculation shows that

$$
\frac{\partial U\left(P_{12}^{ \pm}\right)}{\partial q_{2}}=2 \alpha_{2}\left(P_{12}^{ \pm}\right)
$$

It is noted that $\alpha_{2}\left(P_{12}^{+}\right)>0$ and $\alpha_{2}\left(P_{12}^{-}\right)<0$ for $q_{2}>0$. Therefore, $U\left(P_{12}^{+}\right)$increases and $U\left(P_{12}^{-}\right)$decreases for $q_{2}>0$. Without loss of generality we assume that $q_{2}>0$ in the following discussion. Consequently, the energy barrier becomes $U\left(P_{12}^{-}\right)$, and it decreases when $q_{2}$ increases. Thirdly, the total energy $H_{\infty}$ becomes

$$
H_{\infty}=\left(\alpha_{1}^{0}\right)^{2}+16\left(\alpha_{2}^{0}\right)^{2}+2\left[\frac{h \alpha_{1}^{0}}{2}+\frac{\left(\alpha_{1}^{0}\right)^{2}}{4}+\left(\alpha_{2}^{0}\right)^{2}+e\right]^{2}+2 q_{1}\left(h+\alpha_{1}^{0}\right)+2 q_{2} \alpha_{2}^{0} .
$$


It is noted that $\alpha_{1}^{0}$ and $\alpha_{2}^{0}$ represent the equilibrium positions before any end motion and are dependent upon $q_{1}$ and $q_{2}$. Further calculation shows that

$$
\frac{\partial H_{\infty}}{\partial q_{2}}=2 \alpha_{2}^{0}+4 e \frac{q_{1}-h}{\left(\alpha_{1}^{0}+h\right)^{2}} \frac{\partial \alpha_{1}^{0}}{\partial q_{2}} .
$$

Eqs. (50) and (52) can be used to estimate the effect of $q_{2}$ on $e_{\mathrm{cr}}$. For simplicity, we consider the variation of $H_{\infty}-U\left(P_{12}^{-}\right)$near $q_{2}=0$. We can show that $\alpha_{2}^{0}=0$ and $\partial \alpha_{1}^{0} / \partial q_{2}=0$ when $q_{2}=0$. Therefore,

$$
\left.\frac{\partial\left(H_{\infty}-U\left(P_{12}^{-}\right)\right)}{\partial q_{2}}\right|_{q_{2}=0}=-2 \alpha_{2}\left(P_{12}^{-}\right)>0 .
$$

In other words, $q_{2}$ tends to increase the difference between $H_{\infty}$ over the energy barrier. Therefore, the existence of non-zero $q_{2}$ makes it easier for the arch to snap dynamically and the absolute value of the critical distance $e_{\mathrm{cr}}$ would be smaller.

In Fig. 11 we plot the dangerous zone for $h=3 \sqrt{6}$. The thin lines are for $q_{2}=0$ and have been plotted in Fig. 4(d). The thick lines represent the boundary of the dangerous zone when $q_{2}=10$. To demonstrate the movement of the dangerous zones due to $q_{2}$ we label the corners of the dangerous zones for $q_{2}=0$ and $q_{2}=10$ with unprimed and primed letters, respectively. It is seen that the absolute value of $e_{\text {cr }}$ is smaller for both stretching and compressing processes when $q_{2}=10$. In Fig. 12 we show the history of $\alpha_{1}$ for an arch under stretching with $h=3 \sqrt{6}, q_{1}=10, c=40$, and $e=4$. The arch is in $P_{0}$ position before the prescribed end motion. The damping constant $\mu$ is chosen to be 0.005 . The initial conditions are the same as Eqs. (16) and (17). This loaded arch is represented in Fig. 11 by a black dot, which is in the dangerous zone for $q_{2}=10$ and should be safe for $q_{2}=0$. These predictions are verified in Fig. 12, in which the solid lines and the dashed lines are for $q_{2}=10$ and $q_{2}=0$, respectively. The $\alpha_{1}$ for equilibrium positions $P_{0}, P_{1}^{-}$, and $P_{12}^{-}$for $q_{2}=10$ and $q_{2}=0$ are also plotted as dashed lines for reference.

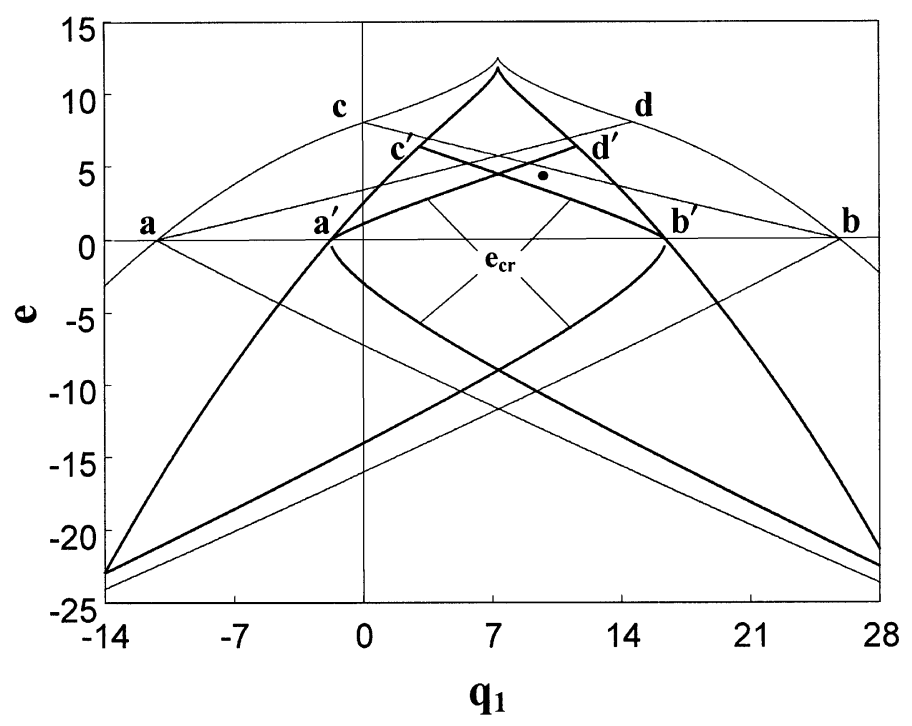

Fig. 11. Dangerous zone for $h=3 \sqrt{6}$. The thin lines and the thick lines are for $q_{2}=0$ and $q_{2}=10$, respectively. The small dot is in safe zone when $q_{2}=0$, but is in dangerous zone when $q_{2}=10$. 

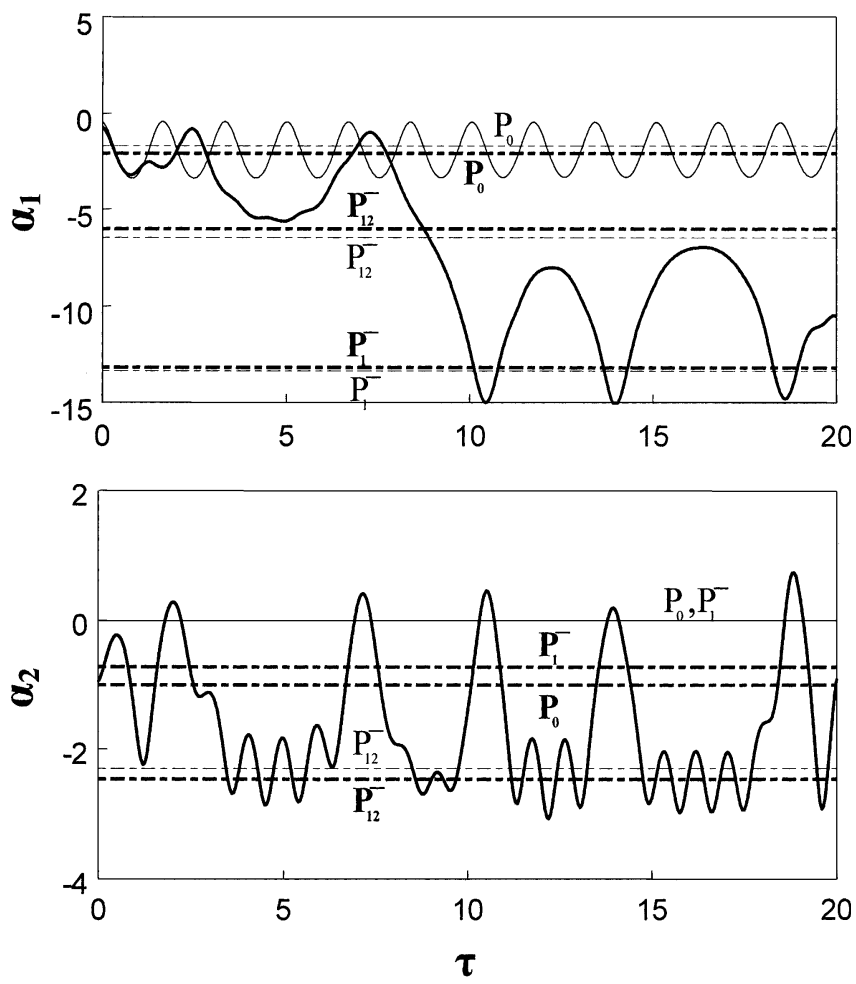

Fig. 12. Response history during stretching for $h=3 \sqrt{6}, q_{1}=10, c=40, e=4$, and $\mu=0.005$. The solid and dashed lines are for $q_{2}=10$ and 0 , respectively.

\section{Conclusions}

In this paper we investigate the effects of lateral loading on the dynamic behaviors of a shallow arch under prescribed end motion. The first harmonic component $q_{1} \sin \xi$ of the lateral load is assumed to be the dominant one, while the effects of higher components such as $q_{2} \sin 2 \xi$ are also discussed in detail. First of all when $q_{2}=0$ some conclusions can be summarized in the following:

(1) The laterally loaded arch may undergo snap-through buckling when the end is stretched quasi-statically. On the other hand, no snap-through is possible when the arch is compressed quasi-statically.

(2) When the end speed of the loaded arch is not negligible, dynamic snap-through may occur in either stretching or compressing process. The dangerous zones for dynamic snap-through can be determined by comparing the energy barrier and the total energy gained by the arch when the end is moved with infinitely large speed.

(3) An arch with ideal initial condition will never undergo dynamic snap-through when it is compressed. On the other hand dynamic snap-through can occur during compressing process when the arch is disturbed at the instant when the end starts to move.

In the case when both $q_{1}$ and $q_{2}$ are non-zero, then some more conclusions can be made in the following:

(4) The equilibrium solutions involve either two or three harmonic modes.

(5) In the case when $q_{1} \neq h$ the original sub-critical pitchfork bifurcation for solutions $P_{12}^{ \pm}$in the bifurcation diagram is destroyed, and new saddle-node bifurcation points are created. The new bifurcation points $e_{j}^{\prime}$ are smaller than the original $e_{j}$ with $q_{2}=0$. 
(6) In the special case when $q_{1}=h$ the original super-critical pitchfork bifurcation in the bifurcation diagram is destroyed, and new saddle-node and super-critical pitchfork bifurcation points are created.

(7) The absolute value of the critical distance $e_{\mathrm{cr}}$ is smaller. That means in order to snap the arch to the other side dynamically, a smaller stretching or compressing distance will do the job if $q_{2} \neq 0$.

\section{Acknowledgement}

The results presented here were obtained in the course of research supported by a grant from the National Science Council of the Republic of China.

\section{References}

Budiansky, B., Roth, R.S., 1962. Axisymmetric dynamic buckling of clamped shallow spherical shells. Collected Papers on Instability of Shell Structures. NASA TN D-1510.

Franciosi, V., Augusti, G., Sparacio, R., 1964. Collapse of arches under repeated loading. ASCE Journal of Structure Division 90, $165-201$.

Fung, Y.C., Kaplan, A., 1952. Buckling of low arches or curved beams of small curvature. NACA Technical Note 2840.

Gjelsvik, A., Bonder, S.R., 1962. The energy criterion and snap buckling of arches. ASCE Journal of Engineering Mechanics Division $88,87-134$.

Hoff, N.J., Bruce, V.G., 1954. Dynamic analysis of the buckling of laterally loaded flat arches. Journal of Mathematics and Physics 32 , 276-288.

Hsu, C.S., 1967. The effects of various parameters on the dynamic stability of a shallow arch. ASME Journal of Applied Mechanics 34, 349-358.

Hsu, C.S., 1968. Stability of shallow arches against snap-through under timewise step loads. ASME Journal of Applied Mechanics 35, 31-39.

Hsu, C.S., Kuo, C.T., Lee, S.S., 1968. On the final states of shallow arches on elastic foundations subjected to dynamical loads. ASME Journal of Applied Mechanics 35, 713-723.

Humphreys, J.S., 1966. On dynamic snap buckling of shallow arches. AIAA Journal 4, 878-886.

Kistler, L.S., Waas, A.M., 1998. Experiment and analysis on the response of curved laminated composite panels subjected to low velocity impact. International Journal of Impact Engineering 21, 711-736.

Kistler, L.S., Waas, A.M., 1999. On the response of curved laminated panels subjected to transverse impact loads. International Journal of Solids and Structures 36, 1311-1327.

Lee, H.N., Murphy, L.M., 1968. Inelastic buckling of shallow arches. ASCE Journal of Engineering Mechanics Division 94, $225-239$.

Lin, J.-S., 2002, Dynamic stability of a shallow arch under prescribed end motion. Master Thesis, Department of Mechanical Engineering, National Taiwan University, Taipei, Taiwan.

Lock, M.H., 1966. The snapping of a shallow sinusoidal arch under a step pressure load. AIAA Journal 4, $1249-1256$.

Onat, E.T., Shu, L.S., 1962. Finite deformation of a rigid perfectly plastic arch. ASME Journal of Applied Mechanics 29 , 549-553.

Patricio, P., Adda-Bedia, M., Amar, M.B., 1998. An elastica problem: instabilities of an elastic arch. Physica D 124, $285-295$.

Roorda, J., 1965. Stability of structures with small imperfections. ASCE Journal of Engineering Mechanics Division 91, 87-106.

Schreyer, H.L., Masur, E.F., 1966. Buckling of shallow arches. ASCE Journal of Engineering Mechanics Division 92, 1-19.

Simitses, G.J., 1973. Snapping of low pinned arches on an elastic foundation. ASME Journal of Applied Mechanics 40, 741-744.

Simitses, G.J., 1990. Dynamic Stability of Suddenly Loaded Structures. Springer-Verlag, New York.

Timoshenko, S.P., 1935. Buckling of flat curved bars and slightly curved plates. ASME Journal of Applied Mechanics 2, 17-20.

Xu, J.-X., Huang, H., Zhang, P.-Z., Zhou, J.-Q., 2002. Dynamic stability of shallow arch with elastic supports-application in the dynamic stability analysis of inner winding of transformer during short circuit. International Journal of Non-Linear Mechanics 37 , 909-920. 